\title{
KING STEPHEN
}





\section{KING STEPHEN \\ II $35-\operatorname{II} 54$}

R. H. C. Davis

University of California Press Berkeley and Los Angeles 1967 
(C) R. H. C. Davis 1967

First publisbed 1967

UNIVERSITY OF CALIFORNIA PRESS

Berkeley and Los Angeles

Library of Congress

Catalogue Card No.: 67-17539 